Rev. Elev. Méd. vét. Pays trop., 1970, 23 (3) : 283-93

\title{
Observations sur les muco-anticorps nasaux des bovins
}

\author{
par A. PROVOST (*) \\ (avec la collaboration technique de Mmes G. DUFAU \\ et N. HASCOUETT, et de M. Z. N'GALDAM)
}

\begin{abstract}
RESUME
Il existe normalement dans le mucus nasal des bovins d'Afrique centrale des substances à activité virucide qui ont le comportement 1mmunologique d'immunoglobulines $\operatorname{IgA}$ et $\mathrm{IgM}$ et possèdent une activité sérologique spécifique. Elles paraissent être d'origine locale et non pas plasmatique ainsi que l'attestent, dans les conditions des expériences, l'absence d'activité antibovipestique du mucus nasal de nombreux veaux à immunité colostrale et de la plupart des bovins adultes vaccinés contre la peste par voie sous-cutanée; inversement, les viroses à tropisme respiratoire supérieur se traduisent par une activité virucide spécifique du mucus nasal. La conséquence pratique est la conservation de la réceptivité des voies aériennes supérieures chez les bovins vaccinés par voie souscutanée, ce qui pourrait avoir d'importantes incidences dans l'épizootiologie de certaines viroses bovines, dont la peste.
\end{abstract}

\section{INTRODUCTION}

C'est avec une certaine surprise que l'on avait observé, lors d'une expérience sur le comportement clinique et immunologique de bovins vaccinés depuis deux ans contre la peste bovine avec des vaccins de cultures cellulaires puis soumis à une infection bovipestique par contamination directe, que le virus contaminant pouvait être retrouvé dans le mucus nasal de certains animaux soumis à cette contamination et restés apparemment en bonne santé; en aucun cas une activité inhibitrice du virus pestique n'avait été mise en évidence dans le mucus nasal (20). Ceci pouvait expliquer cela.

On notera, dans ce qui vient d'être dit, que c'est avec prudence que l'on a parlé "d'éventuelles substances inhibitrices dans le mucus nasal ", sans aucunement inférer qu'il puisse s'agir d'anticorps, ce qui ne pouvait être admis faute de preuves expérimentales.

La situation était tout de même paradoxale, voire inquiétante, et à tout le moins nouvelle, qui semblait indiquer que certains bovins immuns vis-à-vis de la peste bovine pouvaient servir de véhicules du contage par leurs voies nasales supérieures. On aurait pu augurer $a$ priori qu'ils fussent des acteurs inertes dans la contamination car possédant de solides anticorps sériques, anticorps que l'on pouvait s'attendre à retrouver, d'après les connaissances classiques d'immunologie (29), dans certaines sécrétions muqueuses de surface de l'économie, le mucus des voies respiratoires en particulier (13).

Il n'en était rien dans l'expérience qui vient d'être citée et il tombe sous le sens que la question devait être tirée au clair à cause des importantes conséquences théoriques et pratiques qui pouvaient en découler pour l'épizootiologie de certaines viroses bovines.

La recherche de substances virucides dans le mucus nasal de bovins d'Afrique centrale, la démonstration de leur activité neutralisante de certains virus, puis leur caractérisation comme immunoglobulines font l'objet des lignes suivantes. Il a paru préférable d'exposer ainsi les résultats pour mieux suivre le fil de la pensée. 


\section{MATERIEL ET METHODES}

Faute de moyens de caractérisation physicochimiques (ultracentrifugation, chromatographie), les techniques de recherches mises en œuvre ont spécialement été axées sur l'immunologie.

1. Récolte de mucus nasal. Dans les premiers essais, le mucus nasal a été récolté sur écouvillon de coton cardé, ainsi que cela a déjà été dit précédemment (20), après nettoyage du mufle et des naseaux à l'alcool; le mucus était exprimé dans quelques millilitres de solution tamponnée polyantibiotique (pénicilline $100 \mathrm{U} / \mathrm{ml}$, streptomycine $100 \mu \mathrm{g}$, néomycine $50 \mu \mathrm{g}$, kanamycine $25 \mu \mathrm{g}$, fungizone $50 \mu \mathrm{g}$ ) puis homogénéisé à la pipette. On considérait que l'on obtenait une dilution au $1 / 5$, ce qui était dans l'ensemble exact aux erreurs individuelles près; au demeurant, les recherches sur le mucus n'ont été que qualitatives.

Par la suite le mucus a été tout simplement récolté en insérant dans la cavité nasale un tube de verre d'une vingtaine de centimètres de long et de $8 \mathrm{~mm}$ de diamètre, à une extrémité rodée, et adapté sur l'autre extrémité à un tuyau d'aspiration buccale.

On obtient ainsi une certaine quantité de mucus qui est plus exactement mesurable et par là permet de réaliser une dilution précise. Le même mucus, ainsi récolté et non dilué, peut servir aux électrophorèses, aux immunodiffusions et à l'immunofluorescence.

Les dilutions de mucus (dites « mucus» dans la suite du texte) destinées aux séro-neutralisations sont inactivées pendant 30 minutes à $56^{\circ} \mathrm{C}$ pour détruire d'éventuels virus thermosensibles de contamination; elles sont ensuite conservées à $-20^{\circ} \mathrm{C}$ jusqu'à leur utilisation.

2. Sérum sanguin. Classiquement obtenu par ponction veineuse, exécutée chaque fois que cela a été possible en même temps que la récolte de mucus.

3. Techniques sérologiques.

a) Neutralisation. Dilutions de mucus nasal et sérums sont soumis aux tests de neutralisation en cultures cellulaires conduits selon la méthodologie générale de PLOWRIGHT et FERRIS (14) dite à virus constant-anticorps variable. Toutefois, et pour tenir compte de la prédilution du mucus et pour redonner à la réaction plus de sensibilité dans la détection de traces d'éventuels anticorps neutralisants, les réactions ont été effectuées avec seulement $20 \mathrm{DCP}_{\pi 0} / \mathrm{ml}$ des virus sous test (bovipestique, maladie des muqueuses, rhino-trachéitique, parainfluenza 3) au lieu des $200 \mathrm{DCP}_{50}$ habituellement utilisées; on sait en effet qu'il existe une relation linéaire entre les logarithmes des concentrations de virus et d'anticorps dans les réactions de séro-neutralisation (14). Les titres des anticorps sériques ainsi obtenus ne sont donc pas comparables à ceux que l'on a déterminés dans d'autres travaux expérimentaux; en général, ils sont plus élevés par suite de la sensibilité plus grande intentionnellement donnée à la réaction.

b) Inhibition de l'hémagglutination. Elle est conduite pour la recherche des anticorps antiparainfluenza-3 ainsi qu'il a déjà été décrit (19), sur mucus et sérums correspondants.

c) Electrophorèse et immunoélectrophorèse. L'électrophorèse sur papier suit les normes et utilise l'appareillage déjà décrits (21). Mucus et sérums correspondants ont été traités ensemble.

L'immunoélectrophorèse a été, quant à elle, réalisée sur bandes d'acétate de cellulose prédécoupées avec puits et rigoles, et en utilisant lappareillage Millipore ( ${ }^{1}$ ); l'immunsérum révélateur a été préparé localement sur lapin hyperimmunisé avec des sérums de zébus (18) mais a également été utilisé un immunsérum commercial anti- $\gamma$-globulines bovines $\left({ }^{2}\right)$. Il a paru avantageux de faire passer le courant pendant 35 minutes plutôt que 20 comme le préconise le fabricant.

d) Immunofluorescence. Cette technique n'a été utilisée que qualitativement pour caractériser les substances inhibitrices du mucus nasal; elle permet d'apporter une preuve supplémentaire de la nature immunoglobulinique des substances et de leur assigner une spécificité antivirale précise. On a, pour ce faire, utilisé une technique indirecte: les antigènes spécifiques sont représentés par des cultures sur lamelles en tubes de Leighton de cellules

(1) Appareillage Phoroslide. Millipore S.A., 39, rue Chauvelot, 92 Malakoff, France.

(2) Microbiological Associates, 4733 Bethesda Ave nue, Bethesda, Md 20014, U.S.A. 
rénales bovines de première explantation infectées avec les virus de la peste bovine, de la rhinotrachéite infectieuse ou para-influenza 3 ; les lamelles sont fixées dans le méthanol à - 20 $\mathrm{C}$ pendant plusieurs jours, rincées en tampon à $\mathrm{pH} 7,2$, recouvertes de mucus que l'on laisse agir 30 minutes à $37^{\circ} \mathrm{C}$ en atmosphère humidifiée, rincées de nouveau puis "révélées" avec un sérum de lapin conjugué fluorescent anti- $\gamma$ globulines bovines $\left({ }^{3}\right)$ pendant encore 30 minutes à $37^{\circ} \mathrm{C}$, rincées et examinées en lumière ultra-violette (lampe Osram H-200; filtres d'excitation B G 12; filtre d'arrêt 50); des lamelles de cellules non infectées sont incluses comme témoins. Dans chaque essai figure un sérum bovin positif pour l'antigène sous test, à titre de témoin. On n'a procédé à aucune absorption par des poudres d'organes.

\section{RESULTATS}

1. Mise en évidence d'un manque de concordance entre l'existence d'anticorps sériques

(3) Institut Pasteur, 26, rue du Docteur Roux, 75, Paris (15e); ce sérum est dilué au $1 / 40$ lors de l'utilisation. neutralisants et une éventuelle activité inhibitrice du mucus nasal.

a) Dans un essai préliminaire portant sur 57 échantillons de mucus provenant de bœufs adultes vaccinés à plusieurs reprises contre la peste bovine, on n'a découvert d'activité neutralisante du mucus pour le virus pestique que dans trois échantillons seulement. Les 'sérums correspondants possédaient des titres neutralisants $\mathrm{TN}_{50} \geqslant 10^{1.2}$.

b) Lors d'un essai ultérieur sur des prélèvements correspondants de mucus nasal et de sérum de bovins vaccinés contre la peste, on recherche l'activité neutralisante pour le virus pestique et pour le virus de la rhinotrachéite bovine infectieuse, infection largement répandue en Afrique centrale (17) quoique passant souvent inaperçue. Le tableau I rend compte des résultats.

On remarquera que si les anticorps rhinotrachéitiques sont retrouvés dans 75 p. 100 des sérums, chiffre en accord avec un sondage sérologique précédent (17), une activité inhibitrice du mucus n'est présente que dans 2 échantillons sur 12. A noter toutefois, fait important, que lorsque le mucus est inhibant, le sérum correspondant est neutralisant.

TABLEAU $\mathrm{N}^{\circ} \mathrm{I}$

Recherche quantitative des activitês neutralisantes du mucus nasal et du sérum de bovins pour les virus bovipestiques et rhinotrachéitiques.

\begin{tabular}{|c|c|c|c|c|}
\hline \multirow[t]{2}{*}{$\begin{array}{l}\mathrm{N}^{*} \text { des } \\
\text { bovins }\end{array}$} & \multicolumn{2}{|c|}{$\begin{array}{c}\text { Activité } \\
\text { antibovipestique }\end{array}$} & \multicolumn{2}{|c|}{$\begin{array}{l}\text { Activité } \\
\text { antirhinotrachëitique }\end{array}$} \\
\hline & Mucus & Sérum & Mucus & Sérum \\
\hline 5.641 & - & + & + & + \\
\hline 42 & - & + & - & + \\
\hline 43 & - & + & - & - \\
\hline 44 & - & + & - & - \\
\hline 45 & - & + & - & + \\
\hline 46 & - & + & + & + \\
\hline 47 & - & + & - & + \\
\hline 48 & - & + & - & - \\
\hline 49 & - & + & - & + \\
\hline 50 & - & + & - & + \\
\hline 51 & - & + & - & + \\
\hline 52 & - & + & - & + \\
\hline
\end{tabular}

Les signes + et - Indiquent respectivement la prësence ou l'absence d'activité neutrallsante dans les êchantillons. 
Les bovins avaient été vaccinés contre la peste au laboratoire; âgés de 18 mois environ, on ne peut parler pour eux de chute de l'immunité antipestique active, ce qui est reflété par l'existence des anticorps sériques antibovipestiques. Pourtant, on ne trouve pas d'activité neutralisante dans le mucus nasal.

\section{Examen parallèle du mucus nasal et du sérum correspondant de veaux à immunité colostrale.}

La présence d'une activité neutralisante du virus rhinotrachéitique dans les mucus des bovins $\mathrm{n}^{0} 5641$ et $5646 \mathrm{du}$ tableau I devait poser la question de la possibilité du passage des anticorps sériques dans les sécrétions muqueuses, ainsi que cela a été montré chez la souris par FAZEKAS de ST. GROTH (10).

Le problème pouvait être aisément résolu en examinant mucus et sérums de veaux qui, s’ils ont des anticorps sériques dans leur très jeune âge, le doivent au transfert de ceux de leur mère par le colostrum. La comparaison de divers prélèvements pour divers virus pouvait être fructueuse. Elle a été faite pour le virus bovipestique et le virus de la maladie des muqueuses, maladie elle aussi largement répandue en Afrique centrale et pour laquelle un sondage montre que 75 p. 100 des bovins adultes ont des anticorps (16).

\section{Le tableau 2 collige les résultats.}

Le groupement de veaux $\mathrm{n}^{0}$ I était celui d'un marchand de bétail; d'âge moyen relativement avancé, son cas sera discuté plus loin. Le comportement du groupement II est plein d'intérêt. Une remarque préliminaire est à faire quant aux anticorps antipestiques du $\mathrm{n}^{\circ} 5150$ : non recherchés (épuisement du prélèvement), il est vraisemblable qu'ils existaient étant donné l'âge du veau et la présence d'anticorps antimaladie des muqueuses dans son sérum, attestant que sa mère n'était pas en hypo- $\gamma$ globulinémie lors de la première tétée (18). Pour 9 veaux qui possèdent des anticorps sériques antipestiques, on ne retrouve d'activité neutralisante dans le mucus que pour deux d'entre eux ( $\mathrm{n}^{\circ} 5148$ et 5155), tous deux âgés d'un mois. Par contre, aucun veau n'a d'activité inhibitrice dans son mucus nasal pour le virus de la maladie des muqueuses alors que 14 sur 18 possèdent des anticorps sériques neutralisants. Fait saillant, les 2 veaux chez qui on avait trouvé une activité inhibitrice du virus bovipestique dans le mucus n'en possèdent pas pour celui de la maladie des muqueuses bien qu'ayant des $\mathrm{TN}_{50}$ sériques $\geqslant 10^{1,2}$.

Il est aisé de tirer la conclusion que la présence d'une activité inhibitrice dans le sérum n'est pas obligatoirement corrélative de celle du mucus; il ne paraît pas qu'il y ait passage des anticorps sériques dans le mucus nasal au travers de la muqueuse car, gardant à l'esprit la discordance des activités des mucus des veaux 5148 et 5155 , il est bien difficile d'admettre que le transfert muqueux soit sélectif d'une activité neutralisante du sérum plutôt que d'une autre.

On est alors conduit à chercher aux activités inhibitrices des mucus une autre origine. Faisant appel à ce qui est connu en immunologie comparée, deux explications possibles viennent à l'esprit :

- celle d'une activité inhibitrice non spécifique par une substance virucide, du type properdine par exemple; mais alors, étant un mécanisme naturel de défense, on voit mal pourquoi elle serait si irrégulièrement distribuée chez les bovins et surtout pourquoi, arguant toujours du cas des veaux 5148 et 5155 , elle serait active sur le virus pestique et non sur celui de la maladie des muqueuses, virus à structure lipo-protéique de surface comme le premier;

- celle, beaucoup plus plausible, d'une authentique activité neutralisante par anticorps produits in loco au sein des follicules lymphatiques de la muqueuse (26). C'est la thèse qui sera défendue plus bas, en accord d'ailleurs avec ce qui vient d'être récemment démontré chez l'homme $(28,2,4,5,6,7,22,23,25)$.

\section{Exemples de concordance entre la pré- sence d'anticorps neutralisants et l'activité inhi- bitrice spécifique homologue du mucus nasal.}

Si l'hypothèse qui vient d'être énoncée est valable, une infection locale nasale doit laisser plus volontiers sa trace dans le mucus nasal qu'une infection générale. On notera à ce propos que l'infection rhinotrachéitıque évoquée plus haut est un mauvais exemple étant donné le tropisme ambivalent du virus, à la fois respiratoire et génital. Une infection pure- 
TABLEAU $\mathrm{N}^{\circ}$ II

Recherche d'activités neutralisantes dans le mucus nasal et le sêrum de veau

\begin{tabular}{|c|c|c|c|c|c|c|}
\hline \multirow[t]{2}{*}{ Troupeau } & \multirow[t]{2}{*}{ Numéro } & \multirow{2}{*}{$\begin{array}{l}\text { Age } \\
\text { (mois) }\end{array}$} & \multicolumn{2}{|c|}{$\begin{array}{l}\text { Anticorps } \\
\text { antibovipestiques }\end{array}$} & \multicolumn{2}{|c|}{$\begin{array}{l}\text { Anticorps } \\
\text { anti maladie } \\
\text { des muqueuses }\end{array}$} \\
\hline & & & Mucus & Sêrurn & Mucus & Sérum \\
\hline I & $\begin{array}{ll}5 & 129 \\
5 & 130 \\
5 & 131 \\
5 & 132 \\
5 & 133 \\
5 & 134 \\
5 & 135 \\
5 & 136 \\
5 & 137\end{array}$ & $\begin{array}{r}>12 \\
12 \\
8 \\
6 \\
8 \\
12 \\
8 \\
8 \\
12\end{array}$ & $\begin{array}{l}+ \\
+ \\
+ \\
- \\
+ \\
+ \\
+ \\
+ \\
+\end{array}$ & $\begin{array}{r}>1,2 \\
0,9 \\
> \\
1,2 \\
0,3 \\
>1,2 \\
0,6 \\
>1,2 \\
>1,2 \\
0,6\end{array}$ & $\begin{array}{l}+ \\
+ \\
- \\
- \\
- \\
- \\
- \\
- \\
-\end{array}$ & $\begin{array}{r}>1,2 \\
>1,2 \\
0,3 \\
1,2 \\
\mathrm{Tr} \\
>1,2 \\
- \\
>1,2 \\
\\
\mathrm{Tr}\end{array}$ \\
\hline II & $\begin{array}{ll}5 & 138 \\
5 & 139 \\
5 & 140 \\
5 & 141 \\
5 & 142 \\
5 & 143 \\
5 & 144 \\
5 & 145 \\
5 & 146 \\
5 & 147 \\
5 & 148 \\
5 & 149 \\
5 & 150 \\
5 & 151 \\
5 & 152 \\
5 & 153 \\
5 & 154 \\
5 & 155 \\
5 & 156 \\
5 & 157\end{array}$ & $\begin{array}{r}6 \\
4 \\
4 \\
4 \\
4 \\
4 \\
12 \\
4 \\
10 \\
2 \\
1 \\
6 \\
2 \\
10 \\
10 \\
12 \\
7 \\
1 \\
1 \\
2\end{array}$ & $\begin{array}{l}- \\
- \\
- \\
- \\
- \\
- \\
- \\
- \\
- \\
- \\
+ \\
- \\
- \\
- \\
- \\
- \\
- \\
+ \\
- \\
-\end{array}$ & $\begin{array}{c}\mathrm{NE} \\
>1,2 \\
> \\
1,2 \\
- \\
\mathrm{NF} \\
0,9 \\
- \\
0,3 \\
\mathrm{NF} \\
>1,2 \\
>1,2 \\
- \\
\mathrm{NF} \quad(+) \\
\mathrm{NF} \\
- \\
- \\
0,3 \\
1,2 \\
- \\
-\end{array}$ & $\begin{array}{l}- \\
- \\
- \\
- \\
- \\
- \\
- \\
- \\
- \\
- \\
- \\
- \\
- \\
- \\
- \\
- \\
- \\
- \\
-\end{array}$ & $\begin{array}{c}0,9 \\
1,2 \\
>1,2 \\
- \\
0,3 \\
- \\
- \\
1,2 \\
1,2 \\
0,6 \\
1,2 \\
- \\
1,2 \\
1,2 \\
\mathrm{NF} \\
0,3 \\
0,6 \\
>1,2 \\
\mathrm{NF} \\
1,2\end{array}$ \\
\hline
\end{tabular}

Les activités neutralisantes sont exprimées par l'exposant du $\log 10 \mathrm{du}$ titre neutralisant 50 p. 100 (TN 50).

ment respiratoire, et même à participation nasale quasi exclusive comme l'infection par virus parainfluenza $3(27)$, est certainement plus suggestive.

On sait que c'est une virose bovine très largement répandue en Afrique centrale puisque plus de 96,7 p. 100 des bovins hébergent des anticorps spécifiques (19). La recherche des activités inhibantes de l'hémagglutinine parainfluenza 3, réalisée sur 29 échantillons de mucus et de sérums correspondants, montre chez tous la même activité.
Un autre exemple est donné par les résultats des deux colonnes de droite du tableau 3. Une infection locale nasale laisse donc sa trace immunitaire à la fois dans le mucus et le sérum sanguin. Cette trace immunitaire estelle le fait d'immunoglobulines?

\section{Nature de l'activité inhibitrice du mucus nasal.}

a) Existence d'immunoglobulines dans le mucus nasal. Dans un premier temps, celle-ci est aisément mise en évidence par électro- 
phorèse sur papier conduite simultanément pour un sérum et le mucus nasal correspondant. On révèle ainsi l'existence dans le mucus d'une substance qui migre électriquement comme une $\gamma$-globuline sérique. On remarquera (figure 1) que cette substance paraît même être unique dans le mucus, à l'exclusion d'albumine, d'a et de $\beta$-globulines. L'hypothèse d'un simple transfert plasmatique transmuqueux paraît une fois de plus être invalidé.

Que cette substance soit réellement une globuline est prouvé par limmuno-électrophorèse qui ne met en évidence qu'un ou deux arcs de précipitation correspondant à des immunoglobulines (1) comme le montrent les exemples de la figure 2. Des réactions de précipitation en gélose réalisées avec sérums et mucus vis-à-vis d'un sérum de lapin spécifiquement anti $\gamma$-globulines bovines (sérum pour immunofluorescence) le démontrent aussi de manière très simple (figure 3 ), sans toutefois préciser la classe de l'immunoglobuline.

b) Hétérogénéité d'activité des immunoglobulines du mucus nasal.

Le spectre d'activité des immunoglobulines d'un mucus nasal peut être analysé par les techniques d'immunofluorescence indirecte visà-vis d'antigènes connus (cultures cellulaires sur lamelles).

Quelques résultats figurent dans le tableau 3.

On constate, comme dans les exemples précédemment donnés, la rareté de l'existence d'immunoglobulines antibovipestiques dans le mucus nasal des bovins adultes vaccinés, observation qui contraste avec l'existence quasi constante des immunoglobulines antiparainfluenza 3.
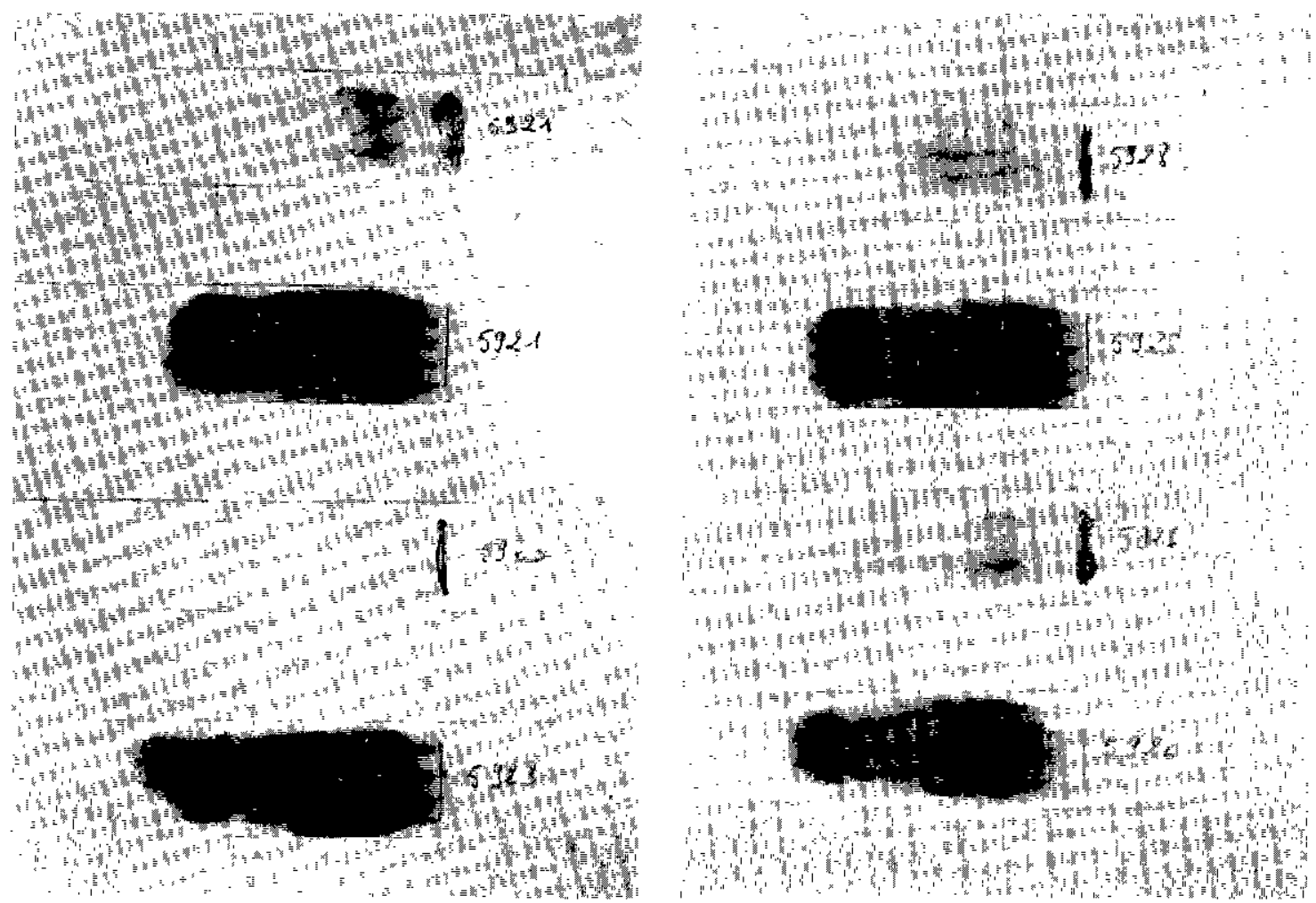

Fig. 1.

Electrophorégrammes sur papier de mucus nasal et des sérums sanguins correspondants Les origines des électrophorégrammes ont été placées sur une même ligne verticale. La simplicité immunologique du mucus est évidente. 
TABLEAU $\mathrm{N}^{\circ}$ III

Analyse par immunofluorescence Indirecte de l'activitê spécifique des imnunoglabulines du mucus nasal de bovins adultes comparée à celle de leur sẻrum sanguin.

\begin{tabular}{|c|c|c|c|c|c|c|}
\hline \multirow{2}{*}{ Numéro } & \multicolumn{2}{|c|}{ Peste bovine } & \multicolumn{2}{|c|}{ Phinotrachélte } & \multicolumn{2}{|c|}{ Parainfluenza 3} \\
\hline & Mucus & Sérum & Mucus & Sêrum & Mucus & Sërum \\
\hline 5511 & - & + & - & - & + & + \\
\hline 5512 & - & + & - & + & - & + \\
\hline 5513 & - & + & - & - & - & + \\
\hline 5514 & - & + & - & + & + & + \\
\hline 5515 & - & + & - & + & + & + \\
\hline 5516 & - & + & - & + & + & + \\
\hline 5517 & + & + & + & + & + & + \\
\hline 5518 & - & + & - & + & + & + \\
\hline 5519 & - & + & - & - & + & + \\
\hline 5520 & ـ & + & - & + & + & + \\
\hline
\end{tabular}

Les signes + et - Indiquent cespectlvement la prẻsence ou l'absence d'une luminosité intense sur des' lames de cultures cellulaires infectées; des témolns appropriés (cultures non infectées, sérums positifs monospécifiques sont introduits en contrôle).
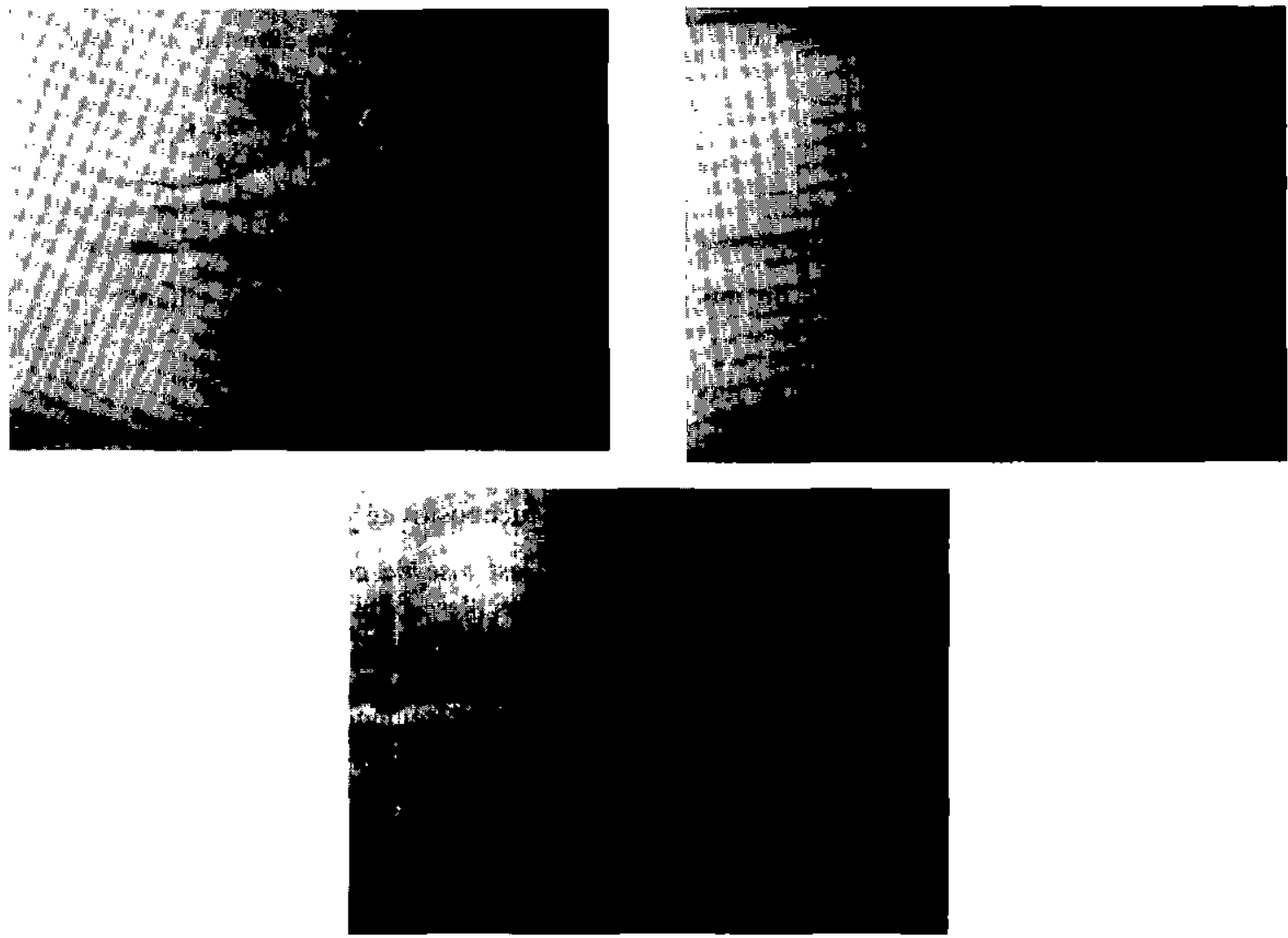

Fig. 2.

Immuno-électrophorégrammes sur acétate de cellulose de mucus nasaux et des sérums sanguins correspondants (*)

(*) Dans les exemples présentés, la révélation a, à dessein, été faite avec un sérum anti $\gamma$-globulınes bovines. Les mucus sont tous en partie supérieure de la rigole centrale, les sérums correspondant en partie inférieure de celle-ci. On voit nettement 2 arcs de précipitation pour les mucus et au moins 3 pour les sérums. 


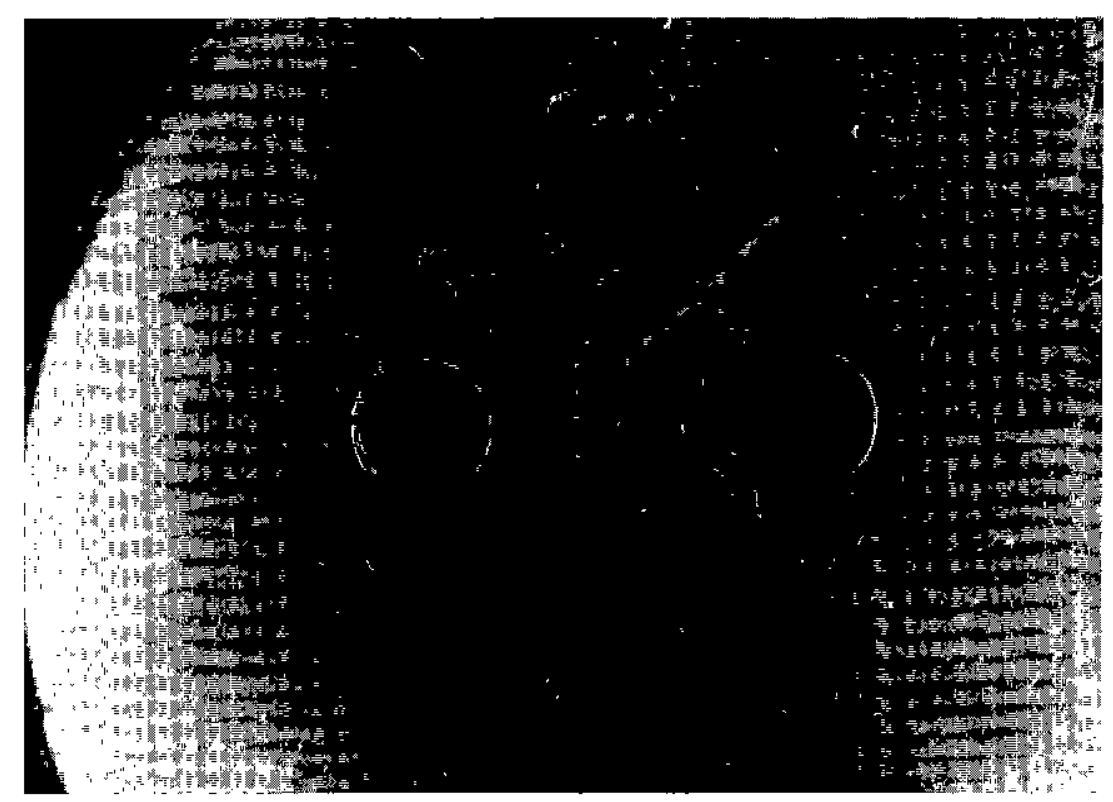

Fig. 3.

Mise en évidence d'immunoglobulines dans le mucus nasal par précipitation-diffusion en gélose

1. Mucus nasal

2. Sérum

\section{Origine des immuno-globulines du mucus nasal.}

Les tableaux 2 et 3 ainsi que la reconnaissance de l'activité inhibant l'hémagglutination du virus parainfluenza 3 laissent à penser que les immunoglobulines du mucus nasal des bovins doivent avoir une production locale et ne résultent pas d'un transfert trans-muqueux de celles du plasma sanguin.

Une expérience simple le prouve. Un groupe de bouvillons de 8 à 12 mois reçoit par voie sous-cutanée environ $250 \mathrm{DCP}_{50}$ de virusvaccin antibovipestique de cultures cellulaires; un autre groupe comparable reçoit ce vaccin par pulvérisation de la muqueuse nasale avec un vaporisateur de parfumerie. On recherche dans les deux groupes la séroconversion antipestique, à la fois dans le sérum sanguin par séroneutralisation en cultures cellulaires et dans le mucus par immunofluorescence indirecte. On a fait figurer ci-dessous un extrait des résultats où ne figurent que des veaux sans anticorps antipestiques colostraux prévacci$\operatorname{naux}\left({ }^{4}\right)$.
3. Antisérum précipitant antizébu

4. Antigammaglobuline de bœuf
Immunisés par voie sous-cutanée 12 Séropositivité postvaccinale 12 Mucopositivité postvaccinale 4

Immunisés par voie pernasale 12 Séropositivité postvaccinale 10

Mucopositivité postvaccinale $\quad 10$

Il est aisé de constater qu'alors que presque tous les bouvillons vaccinés par voie nasale possèdent après vaccination des anticorps dans leurs mucus et dans leurs sérums, quelques-uns seulement de ceux vaccinés par voie sous-cutanée ont des muco-anticorps nasaux antibovipestiques.

\section{DISCUSSION}

Les résultats ci-dessus exposés laissent peu de doutes quant à la réalité de l'existence d'immunoglobulines à la surface des voies

(4) Ces résultats paraîtront in extenso dans un article à venir. 
respiratoires supérieures des bovins et plus spécialement des cavités nasales. Il n'y a là, certes, rien qui doive spécialement surprendre en physiologie comparée, puisque ces anticorps ont été signalés chez l'homme; toutefois, étant donné les circonstances où l'on avait été amené à se poser la question de leur existence (on se rappelle qu'un virus pestique avait été isolé chez les bovins vaccinés contre la peste), il a paru sage de ne pas tenir compte des enseignements obtenus dans l'espèce humaine et de reprendre le problème sous l'angle de l'immunité locale vis-à-vis de quelques viroses bovines.

La présence très inconstante dans le mucus nasal des veaux d'anticorps d'origine colostrale existant au demeurant dans leur sérum, l'existence plus régulière par contre d'un anticorps neutralisant pour un virus à tropisme respiratoire pur, ont alors conduit à émettre l'hypothèse d'une sécrétion locale des immunoglobulines à défaut de leur transport transmuqueux venant du plasma sanguin.

Il est difficile de préciser la nature exacte de ces immunoglobulines.

D'après les immuno-électrophorégrammes, on est tenté de les assimiler à des globulines IgA par suite de l'emplacement de leur migration bien que sur plusieurs immuno-électrophorégrammes on ait également pu remarquer des arcs de précipitation assimilables à des IgM; c'est avec prudence qu'est avancée cette assertion qui contredit la thèse d'AALUND (1) sur l'absence d'IgA chez les bovins au bénéfice des seules IgG et IgM mais qui se trouve en accord avec l'opinion d'autres auteurs (12). Sans moyens locaux de caractérisation physicochimiques, il est difficile d'approfondir l'observation, mais on conçoit l'intérêt de ces recherches.

Ce qui pratiquement paraît être important dans les constatations ci-dessus rapportées, est l'existence d'anticorps nasaux pour quelques maladies à tropisme respiratoire. Le cas est particulièrement net pour l'infection parainfluenza 3. Il nous paraît l'être aussi pour la peste bovine. Dans cette virose, la voie aérienne supérieure est la porte d'entrée, à l'exclusion de la voie digestive inefficace, ainsi que cela a été pressenti puis démontré (24). La comparaison des résultats des tableaux 1 et 3 et de la première partie du tableau 2 est fructueuse; on y constate que des bouvillons "tout-venant", achetés non vaccinés puis l'ayant été au laboratoire, ne possèdent pas de muco-anticorps nasaux spécifiques; cependant 8 sur 9 des bouvillons d'un marchand de bétail, animaux eux-mêmes non vaccinés et qui pourtant, d'après leur âge, devraient être séronégatifs pour la peste, possèdent à la fois séro et muco-anticorps. Ne peut-on être tenté de voir là une immunisation naturelle contre la peste, effectuée peut-être sous le couvert d'une immunité colostrale évanescente, qui se serait établie lors d'une infection occulte par voie aérienne, similaire à celle qui s'établit pour l'infection parainfluenza 3 ?

L'expérience relatée dans le chapitre 5 montre la réceptivité de la voie aérienne supérieure au contage bovipestique (dans le cas présent, vaccinal) qui laisse sa trace immunitaire à la fois dans le sérum et le mucus nasal. On est dès lors conduit à penser qu'en nombre de cas la vaccination antibovipestique effectuée par voie sous-cutanée "court-circuite » la muqueuse nasale au regard de limmunité engendrée et la laisse réceptive à l'infection. Il est à noter qu'a priori, dans cette proposition, on ne fait pas état des cellules qui peuvent être réceptives, épithéliales ou lymphoïdes, regardant la muqueuse nasale comme un tout. Les implications qu'elle recèle ont été développées par ailleurs en ce qui concerne l'épizootiologie actuelle de la peste bovine (15). Pour le sujet qui nous occupe, on notera qu'elle donne l'explication des faits observés dans un essai antérieur (20), faits qui ont motivé la série d'observations ici rapportées et qu'elle se trouve en parfait accord avec ce qu'enseigne l'expérience.

Si l'on se place sur le terrain de la pathologie comparée, la conservation de la réceptivité de la porte d'entrée naturelle dans les viroses après vaccination sous-cutanée a été maintes fois observée et il peut paraitre surprenant qu'on ne l'ait jamais suspectée dans la peste bovine. C'est ainsi que pour le virus de la rougeole le cas est parfaitement établi tant chez le singe (9) que chez l'enfant vacciné avec un virus-vaccin vivant atténué; c'est vrai également d'un autre myxovirus, le virus parainfluenza $I(22,23)$ pour lequel la vaccination par voie sous-cutanée ne suffit pas pour prévenir l'infection nasale. En médecine vétérinaire 
et restant dans le domaine des myxoviroses, on peut citer les exemples de la vaccination du mouton avec le virus parainfluenza 3 qui n'empêche pas l'excrétion nasale du virus d'épreuve inoculé par cette voie (11) ou encore celui de la vaccination locale dans la maladie de Newcastle qui se montre opérante contre la contamination par aérosol à défaut de la voie intramusculaire inopérante (3). C'est aussi le cas dans la péripneumonie où les anticorps sériques après vaccination sous-cutanée ne sont pas retrouvés dans le mucus nasal (8).

Des observations antérieurement faites, des expériences actuelles et des enseignements tirés d'autres maladies, se dégage ainsi la notion d'immunité locale nasale dans la peste bovine. Il est dès lors tentant d'essayer la vaccination par cette voie qui devrait permettre d'atteindre tous les tissus réceptifs et, partant, de couper les relais de réplication du virus bovipestique dans la nature que peuvent constituer les récepteurs des voies aériennes supérieures de bovins par ailleurs correctement vaccinés par voie sous-cutanée.

I.E.M.V.T., Laboratoire de Recherches vétérinaires de Farcha, Fort-Lamy, Tchad.

\section{SUMMARY}

\section{Observations on nasal muco-antibodies in cattle}

Virulicidal substances which are normally existing in the nasal mucus of cattle in central Africa have been shown to possess the immunological behaviour of IgA and IgM immunoglobulins and display specific serological activity. They seem to have a local, not plasmatic, origin as it is attested in the reported experiments by the lack of rinderpest activity in the nasal mucus of calves with colostral antibodies and in most of subcutaneously rinderpest-vaccinated adult cattle; conversely, viral infections with upper respiratory tract tropism display specific virulicidal activity in the nasal mucus. It follows that in subscutaneously-vaccinated cattle the upper respiratory tract remains receptive to local infection, which could have important consequences in the epizootiology of viral diseases, namely rinderpest.

\section{RESUMEN}

\section{Observaciones sobre los mucoanticuerpos nasales de los bovinos}

El en mucus nasal de los bovinos de Africa central naturalmente existen substancias activas contra los virus que tienen el comportamiento immunologico de inmunoglobulinas IgA e IgM y una actividad serologica específica. Parecen ser de origen local y no del plasma como lo comproba, en las condiciones de las experiencias, la falta de actividad antibovipestica del mucus nasal de numerosos terneros inmunizados por el calostro y de la mayor parte de los bovinos adultos vacunados contra la peste por via subcutánea; inversamente, las enfermedades a virus con tropismo respiratorio superior se manifiestan por una actividad contre el vurus específica del mucus nasal. La consecuencia práctica es la conservación de la receptividad de las vias aéreas superiores en los bovinos vacunados por via subcutánea, lo que podria tener importantes incidencias en la epizootiologia de ciertas enfermedades a virus de los bovinos, de las cuales la peste.

\section{BIBLIOGRAPHIE}

1. AALUND (O.), «Heterogeneity of ruminant immunoglobulins ", Copenhague, Munksgaard, 1968.

2. ARTENSTEIN (M.S.), BELLANTI (J. A.) et BUESCHER (E. L.), *Identification of the antiviral substances in nasal secretions », Proc. Soc. exp. Biol. Med., 1964, 117, 558-564.

3. BEARD (C. W.) et EASTERDAY (B. C.), « The influence of the route of administration of Newcastle disease virus on host response. I. Serological and virus isolation studies $", J$. infect. Dis., 1967, 117, 55-61.

4. BELLANTI (J. A.), "Role of local gamma-AImmunoglobulins in immunity $», A m . J . D i s$. Child,. 1968, 115, 239-246.

5. BELLANTI (J.A.) et ARTENSTEIN (M.S.), « Mechanisms of immunity and resistance to virus infections », Pediatric Clinics N. Am., 1964, 11, 549-561. 
6. BELLANTI (J. A.), ARTENSTEIN (M.S.) et BUESCHER (E. L.), "Characterization of virus neutralizing antibodies in human serum and nasal secretions », J. Immun., 1965, 94, 344-351.

7. BRANDTZAEG (P.), FJELLANGER (I.) et GJERULDSEN (S. T.), « Localization of immunoglobulins in human nasal mucosa ", Immunoch., 1967, 4, 56-60.

8. DAVIES (G.), « The examination of nasal mucus for antibodies to Mycoplasma mycoides", Vet. Rec., 1969, 84, 417.

9. ENDERS (J. F.) et PEEBLES (T. C.), « Propagation in tissue cultures of cytopathogenic agents from patients with measles n, Proc. Soc. exp Btol. Med., 1954, 86, 277-286.

10. FAZEKAS de ST. GROTH (S.). Cité par PIERCE (A. E.), référence $\mathrm{n}^{*} 13$.

11. GILMOUR (N.J. L.), DRYSDALE (A.), STEVENSON (R. G.), et al., "Reaction of young adult sheep to vaccination and infection with myxovirus parainfluenza 3 », J. comp. Path., 1968, 78, 463-468.

12. PAN (I. C.), KAPLAN (A. M.), MORTER (R. L.) et al., * Spectrum of ovine immunoglobulins", Proc. Soc. exp. Biol. Med., 1968, 129, 867-870.

13. PIERCE (A. E.), "Specific antibodies at mucous surfaces », Vet. Rev. Annot., 1959, 5, 17-36.

14. PLOWRIGHT (W.) et FERRIS (R. D.), « Studies with rinderpest virus in tissue culture. III. The stability of culture virus and its use in virus neutralization tests », Arch. ges. Virusf., 1961, 11, 516-533.

15. PROVOST (A.), «Réflexions sur l'épizootiologie de la peste bovine en Afrique centrale», Communication Colloque O.C.A.M. sur l'élevage, FortLamy, 8-14 décembre 1969.

16. PROVOST (A.), BOGEL (K.), BORREDON (C.) et MAURICE (Y.), « La maladie des muqueuses en Afrique centrale; observations cliniques et épizootiologiques», Rev. Elev. Méd. vet. Pays trop., 1967, 20, 27-49.

17. PROVOST (A.) et BORREDON (C.) \& Infectious kerato-conjonctivitis in cattle », Vet. Rec., 1965, 77, 1568.

18. PROVOST (A.), BORREDON (C.) et QUEVAL (R.), «Une hypogammaglobulinémie essentielle des bovins d'Afrique centrale, cause d'erreur dans les enquêtes sérologiques », Rev. Elev. Méd. vét. Pays trop., 1965, 18, 385-393.

19. PROVOST (A.), BORREDON (C.), QUEVAL (R.) et MAURICE (Y.), « Enquête sur l'infection des bovidés par le virus para-influenza 3 en Afrique centrale", Rev. Elev. Méd. vét. Pays trop., 1967, 20, 51-59.

20. PROVOST (A.), MAURICE (Y.) et BORREDON (C.), «Comportement clinique et immunologique lors de contamination bovipestique de bovins vaccinés depuis plusieurs années contre la peste bovine avec des vaccins de culture cellulaire $\$, R e v$. Elev. Méd. vét. Pays trop., 1969, 21, 4, 453-64.

21. QUEVAL (R.), "Contribution à l'étude quantitative des protéines sériques du zébu arabe du Tchad », Rev. Elev. Méd. vét. Pays trop., 1959, 12, 293-296.

22. SMITH (C. B.), BELLANTI (J. A.) et CHANOCK (R. M.), «Immunoglobulins in serum and nasal secretions following infection with type I parainfluenza virus and injection of inactivated vaccines », J. Immun., 1967, 99, 133-141.

23. SMITH (C. B.), PURCELL (R. H.), BELLANTI (J. A.) et CHANOCK (R. M.), « Protective effect of antibody to parainfluenza type I virus », New Engl, J. Med., 1966, 275, I, 145.

24. TAYLOR (W. P.), PLOWRIGHT (W.), PILLINGER (R.) et al., "Studies on the pathogenesis of rinderpest in experimental cattle. IV. Proliferation of the virus following contact infection $», J$. Hyg. (Camb.), 1965, 63, 497-506.

25. TOMASI (T. B.) et BIENEWSTOCK (J.), "Secretory Immunoglobulins », Adv. Immunology, 1968, 9. 2-96.

26. TRAUTMANN (A.) et FIEBIGER (J.), « Fundamentals of the histology of domestic animals », Ithaca, U.S.A., Comstock Publishing Associates, 1952.

27. VAN DER MAATEN (M. J.), «Immunofluorescent studies of bovine parainfluenza 3 virus. II. Experimentally infected calves», Canad. J. comp. Med., 1969, 33, 141-147.

28. "Viral infections and local IgA ", New. Engl. J. Med., 1969, 280, 666-667.

29. WILSON (G. S.) et MILES (A. A.), « Topley and Wilson's principles of bacteriology and immunity $», 5^{e}$ éd., London, E. Arnold Itd, 1964. T. II. pp. 1471-73. 